\title{
PROPOSAL FOR A NEW GENERAL $V(\lambda)$ MISMATCH INDEX
}

\author{
Ferrero, A., Velázquez, J.L., Pons, A., Campos, J. \\ Instituto de Óptica "Daza de Valdés" (IO-CSIC), Agencia Estatal CSIC, Madrid, SPAIN \\ alejandro.ferrero@csic.es
}

DOI $10.25039 / \times 44.2017 .0 P 10$

\begin{abstract}
The general $V(\lambda)$ mismatch index, $f_{1}$, was defined by CIE for a general description of the photometric performance of photometers. This index is widely-used in Photometry, and it is very relevant for selecting photometers requiring minimal corrections under different illuminants in photometric measurements. It quantifies the mismatch between the spectral responsivity of a photometer and $V(\lambda)$. However, we found that this index only correlates well with the photometer photometric performance when illuminants with narrow spectral distributions are used. We propose an alternative general $V(\lambda)$ mismatch index, based on the remaining variation of the relative spectral responsivity with respect to $V(\lambda)$ once the highfrequency variation is filtered out. This proposed alternative index correlates much better than $f_{1}{ }^{\prime}$ with the empirical photometric performance of seven photometers when phosphor-based LEDs and blackbody illuminants are considered.
\end{abstract}

Keywords: Photometry, general $V(\lambda)$ mismatch index

\section{Introduction}

The photometric performance of Illuminance and Luminance Meters (hereafter, just photometers) should be adequately assessed by providing quality indices. These indices are defined by the Commission Internationale de I'Eclairage (CIE) in the International Standard CIE S 023/E:2013 [CIE 2013], and provide information about the general $V(\lambda)$ mismatch, the UV or IR responses, the cosine response, the linearity, the temperature dependence, the spatial non-uniform response, and others. Among them, the general $V(\lambda)$ mismatch index, $f_{1}$, is widely-used in Photometry, because it is very important for selecting photometers requiring minimal corrections under different illuminants in photometric measurements.

The spectral responsivity of a photometer should match the spectral luminous efficiency function for photopic vision, $V(\lambda)$ [CIE 2004], in order to avoid using correction factors of the luminous responsivity according to the spectral power distribution (SPD) of the source under measurement. However, this is never the case. For general lighting conditions -in particular, white-light light sources- the quality of the spectral mismatch is expressed by the general $V(\lambda)$ mismatch index, $f_{1}{ }^{\prime}$, defined as:

$$
f_{1}^{\prime}=\frac{\int_{380 n m}^{780}\left|s_{\text {rel }}^{*}(\lambda)-V(\lambda)\right| \mathrm{d} \lambda}{\int_{380 \mathrm{~nm}}^{780 \mathrm{~nm}} V(\lambda) \mathrm{d} \lambda}
$$

where $s_{\text {rel }}^{*}(\lambda)$ is the normalized spectral responsivity function: 


$$
S_{\text {rel }}^{*}(\lambda)=S_{\text {rel }}(\lambda) \cdot \frac{\int_{380 ~}^{38 m} S_{\mathrm{A}}(\lambda) V(\lambda) \mathrm{d} \lambda}{\int_{380 \mathrm{~nm}}^{780 \mathrm{~nm}} S_{\mathrm{A}}(\lambda) S_{\text {rel }}(\lambda) \mathrm{d} \lambda}
$$

$S_{A}$ is the spectral power distribution of the CIE Standard Illuminant $A$, and $S_{\text {rel }}(\lambda)$ is the relative spectral responsivity of the photometer

This index is quite reasonably defined to quantify the mismatch between the spectral responsivity of a photometer and $V(\lambda)$. But, this does not mean, as we will show in this contribution, that this index is well-correlated with the photometric performance of photometers, understanding that a photometer has a good photometer performance if its luminous responsivity has small dependence on the spectral distribution function of the source to be measured. $f_{1}{ }^{\prime}$ is not enough to describe the photometric performance of a photometer, since, as we will show, it also depends on the spectral power distribution of the relative difference between $V(\lambda)$ and the relative spectral responsivity of the photometer, and not only on the accumulated difference across the spectral range, which $f_{1}{ }^{\prime}$ provides. In this contribution we will propose an alternative to the so-called general $V(\lambda)$ mismatch index, which consider the spectral distribution of the difference.

\section{Definition of a new general $V(\lambda)$ mismatch index $f_{1}{ }^{\prime \prime}$}

We can quantify the photometric performance of a photometer for measuring a given source $Z$ of spectral power distribution $S_{Z}(\lambda)$ as the relative difference between the reading of the photometer and the reading that it would have if its relative spectral responsivity was exactly $V(\lambda)$. We denote this relative difference as $\delta s v z(\lambda)$, because it corresponds with a relative difference between the luminous responsivities $(s v)$ when using as illuminant $Z$ :

$$
\delta s_{V, Z}=\left|\frac{\int_{\lambda_{\min }}^{\lambda_{\max }} S_{\mathrm{Z}}(\lambda)\left[S_{\mathrm{rel}}^{*}(\lambda)-V(\lambda)\right] \mathrm{d} \lambda}{\int_{360 \mathrm{~nm}}^{830 \mathrm{~nm}} S_{\mathrm{Z}}(\lambda) V(\lambda) \mathrm{d} \lambda}\right|
$$

where the lower and upper integration limits $\left(\lambda_{\min }, \lambda_{\max }\right)$ cover the entire range where $S_{\text {rel }}^{*}(\lambda)$ has non-zero values. Notice that here a perfect photometric performance is null, and it can be achieved if $s_{\text {rel }}^{*}(\lambda)=V(\lambda)$. It would be almost perfect if the illuminant $\mathrm{Z}$ is CIE Standard Illuminant A, since $S_{\text {rel }}(\lambda)$ was normalized using $S_{A}$ as reference (Eq. (2).

We assume that the values of a general $V(\lambda)$ mismatch index for general lighting should be highly correlated with the average of $\delta s_{V, Z}\left(\left\langle\delta s_{V}\right\rangle\right)$ when averaging values corresponding with illuminants $Z$ used in general lighting.

If we compare the index $f_{1}{ }^{\prime}$ defined in Eqs. (1) and (2) with $\delta s_{V, z}$ in Eq. (3), we see an important difference: in $f_{1}$ ', the absolute value of the difference is taken before integrating with respect to the wavelength, whereas, in $\delta s_{V, Z}$, after integrating. Thus, absolute differences at every wavelength are accumulated in $f_{1}{ }^{\prime}$, whereas they are compensated in $\delta s_{V, Z}$. It involves a crucial difference: In the case of $f_{1}$, negative or positive deviations obtained at every wavelength count in the same way to its final value, whereas, for $\delta s_{V, Z}$, a negative deviation 
and a positive deviation within a narrow spectral range have a higher likelihood to be compensated than if they were negative and positive differences at two well separated spectral regions, because the source radiance values are going to be more different with a higher probability in the latter case. This consideration is subtle but crucial, since, as a consequence, not only the integral value but also the spectral distribution of the relative difference $S_{\text {rel }}^{*}(\lambda)-V(\lambda)$ becomes relevant to assess the photometric performance of a photometer. More specifically, it seems adequate to analyze this difference in terms of spectral frequencies $v_{\lambda}$ (defined analogously to the temporal frequency in a temporal signal). A priori, according to the previous considerations, a photometer with a fast variation of the $S_{\text {rel }}^{*}(\lambda)-V(\lambda)$ with respect to the wavelength would have a better photometric performance than a photometer with slow variation. Based on this, we propose in the following lines an alternative general $V(\lambda)$ mismatch index for general lighting, which we denote $f_{1}$ ".

We define the quantity relative spectral mismatch of a photometer as:

$$
\delta_{\mathrm{s}}(\lambda)=\frac{\bar{s}_{\mathrm{rel}}(\lambda)-V(\lambda)}{\int_{360 \mathrm{~nm}}^{830 \mathrm{~mm}} V(\lambda) \mathrm{d} \lambda}
$$

being $\bar{S}_{\text {rel }}(\lambda)$ a normalized relative spectral responsivity, given as:

$$
\bar{s}_{\text {rel }}(\lambda)=s_{\text {rel }}(\lambda) \cdot \frac{\int_{380 n m}^{780 n m} V(\lambda) \mathrm{d} \lambda}{\int_{380 n m}^{780 n m} s_{\text {rel }}(\lambda) \mathrm{d} \lambda}
$$

It is similar to Eq. (2), but in this case equienergetic illuminant is used instead of $A$.

Then, the proposed general $V(\lambda)$ mismatch index is expressed as:

$$
f_{1}^{\prime \prime}=\sqrt{2 \int_{0}^{v_{\lambda c}}\left|\hat{\delta}_{\mathrm{s}}\right|^{2} \mathrm{~d} v_{\lambda}}
$$

where $\hat{\delta}_{s}$ is the Fourier Transform of $\delta_{s}(\lambda)$, and $v_{\lambda, c}$ is a cut-off spectral frequency. The factor 2 is introduced to account for the two symmetric sides of the Fourier Transform (singlesided amplitude spectrum). With this definition, the proposed index represent the standard deviation of $\delta_{s}(\lambda)$ once the variations at spectral frequencies higher than $v_{\lambda, c}$ were filtered out, unlike $f_{1}$ ' which consider the variation regardless the spectral frequency.

To complete this definition, the value of $v_{\lambda, c}$ must be determined, and it should depend on the general shape of the spectral distributions of the illuminants to be included in the "general lighting". For this determination, we selected a set of 18 illuminants, divided in two subsets. The first subset is composed of 9 phosphor-based LEDs illuminants ( $F$ LED), with CCT of around $2500 \mathrm{~K}, 2700 \mathrm{~K}, 3000 \mathrm{~K}, 3500 \mathrm{~K}, 4000 \mathrm{~K}, 4500 \mathrm{~K}, 5000 \mathrm{~K}, 5700 \mathrm{~K}$ and $6500 \mathrm{~K}$, which correspond with the categories defined by ANSI ANSLG C78.377-2011 [ANSI 2015]. The second subset is composed of 9 blackbody illuminants (BB), distributed with the same colour temperatures than the CCTs quoted for the F LED subset. We used these illuminants, in combination with seven photometers of known spectral responsivities with $f_{1}{ }^{\prime}$ values between 
0.009 and 0.025 , to obtain an average value of $\delta s_{V, Z}$. The spectral curves $\delta_{s}(\lambda)$ of the studied photometers are given in Figure 1, together with their names and their values for $f_{1}$. We can observe that LMT1 is very similar that LMT2 (in shape and $f_{1}$ ' index), and that the same can be said about 130TH and PO143. However, we must notice that 130TH and PO143 have a quite different empirical photometric performance, $\left\langle\delta s_{V}\right\rangle$.

As we said before, the values of a general $V(\lambda)$ mismatch index for general lighting would be highly correlated with the average of $\delta s_{V, Z}\left(\left\langle\delta s_{V}\right\rangle\right)$ [Eq. (3)] when using as illuminants $Z$ those used in general lighting. Therefore, we determined the value of $v_{\lambda, c}$, as the value that better correlation provide between $\left\langle\delta s_{V}\right\rangle$, when calculated using the 18 illuminants before mentioned, and $f_{1}{ }^{\prime \prime}\left[\right.$ Eq. (6)]. The selected value for $v_{\lambda, c}$ was $0.003 \mathrm{~nm}^{-1}$.
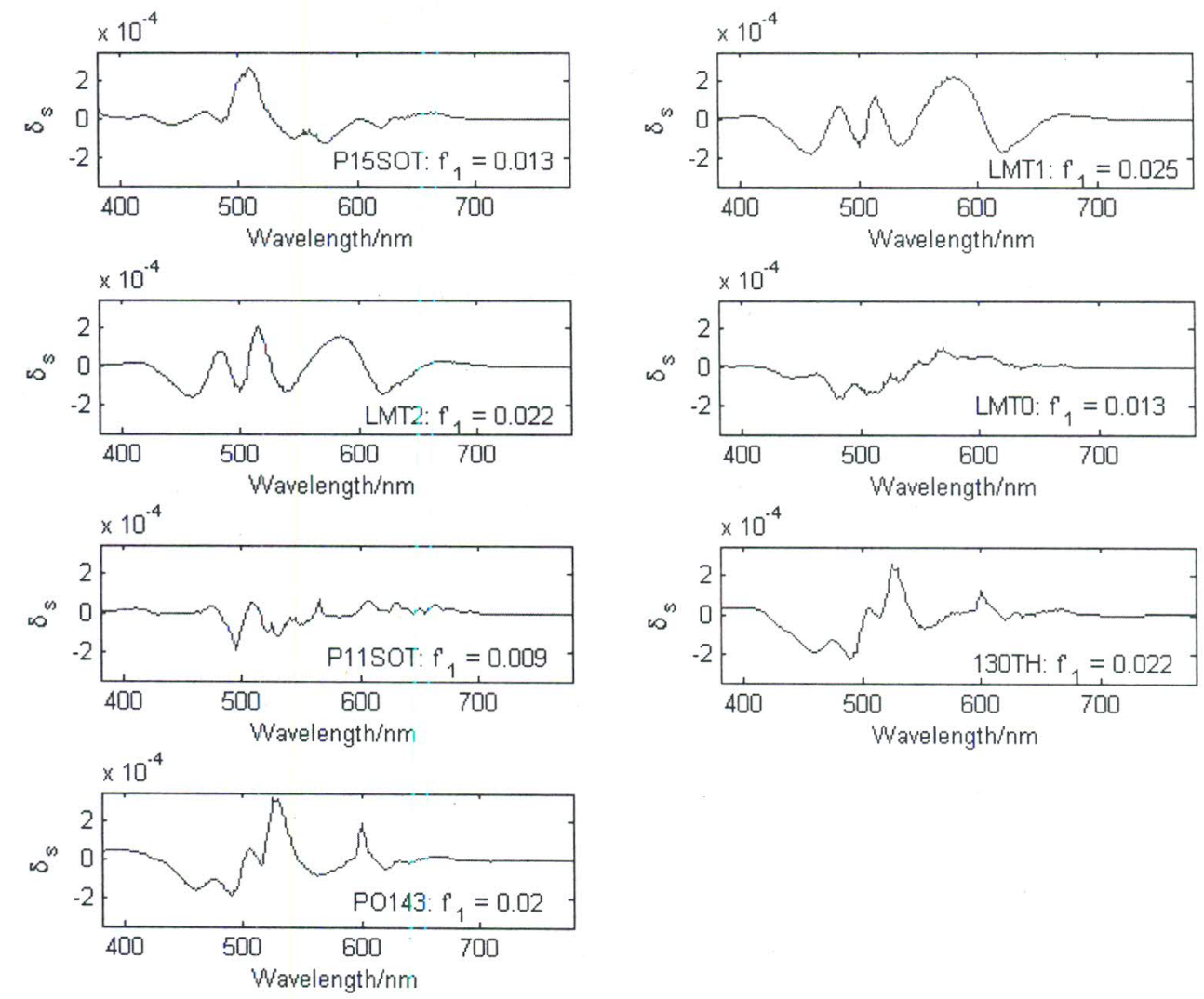

Figure 1 - Spectral curves $\delta_{\mathrm{s}}(\lambda)$ of the seven photometers used to determine the cut-off frequency, $v_{\lambda, c}$

\section{Discussion}

In order to evaluate the improvement of the proposed index $f_{1}$ " with respect the accepted index $f_{1}{ }^{\prime}$, we studied their dependence on $\left\langle\delta s_{V}\right\rangle$, for the seven photometers and when it is calculated using four different kinds of illuminants: 1) The BB subset, 2) the F LED subset, 3) 
the set BB + F LED used to determine $v_{\lambda, c}$, and 4 ) a set including just three LEDs (R, G and $B)$. This dependence is given in Figure 2 and Figure 3 for $f_{1}{ }^{\prime \prime}$ and $f_{1}{ }^{\prime}$, respectively.
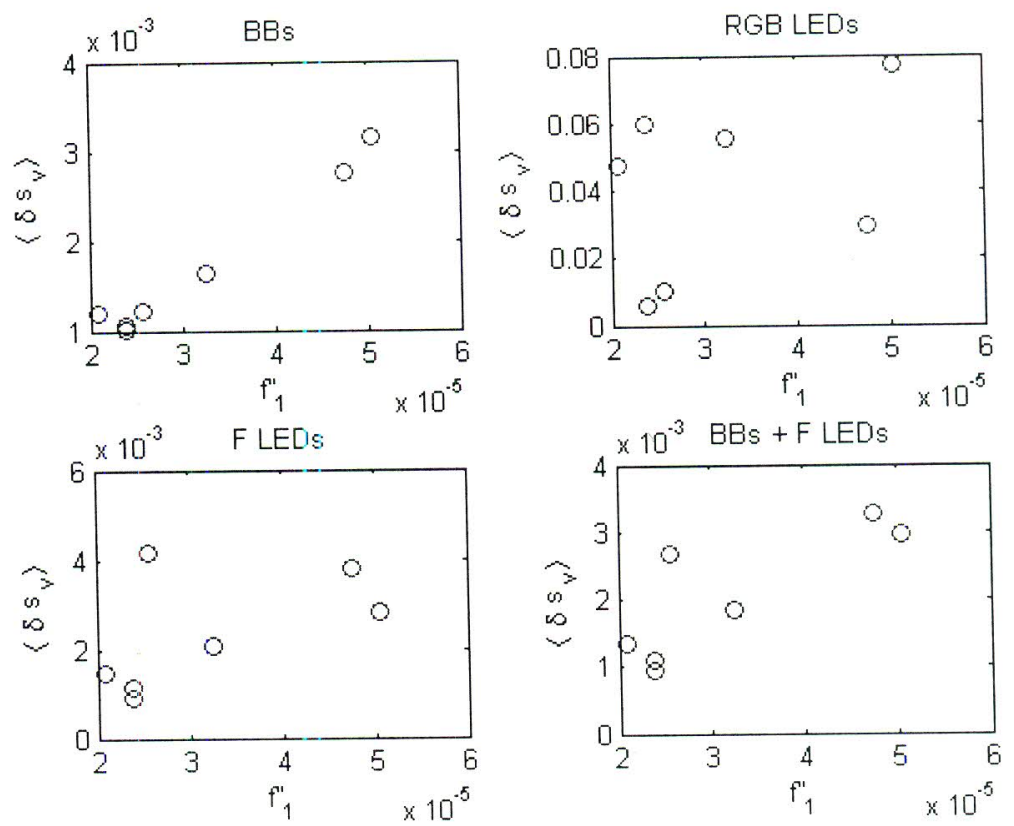

Figure 2 - Relation between $f_{1}$ " and the empirical photometric performance when the latter was calculated using 9 blackbody illuminants (top left), 9 phosphor-based LEDs (bottom left), 3 RGB LEDs (top right), and 9 blackbody illuminants together with 9 phosphor-based LEDs (bottom right)
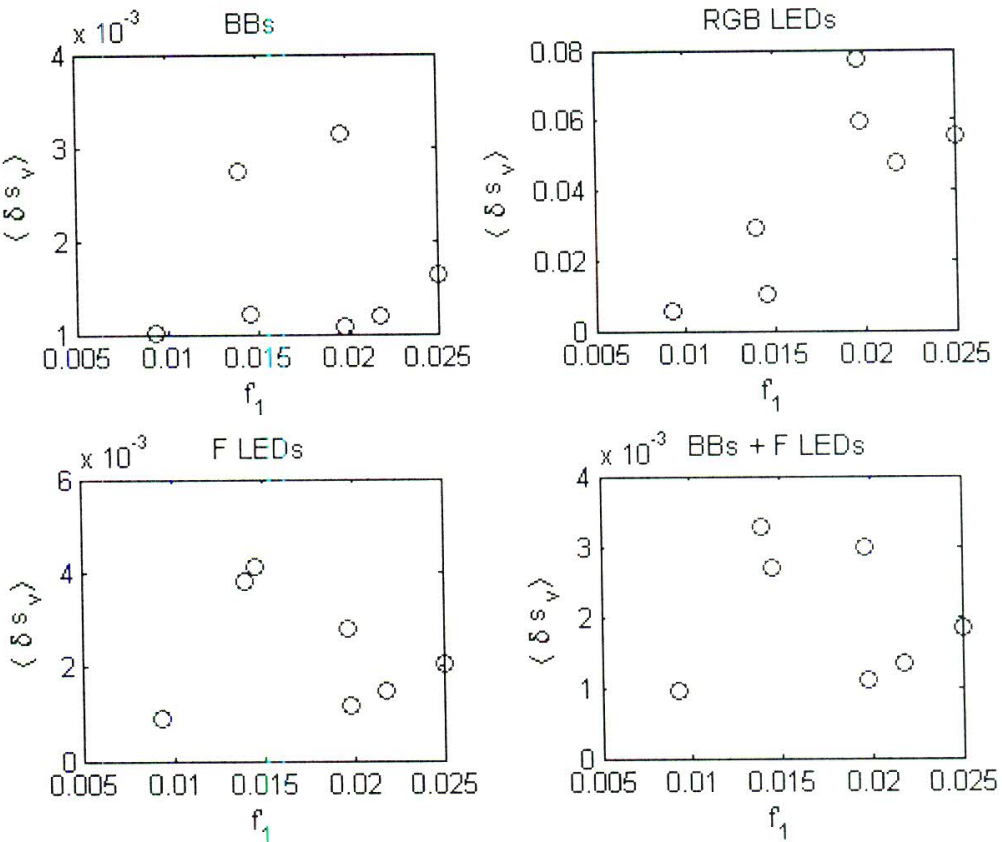

Figure 3 - Relation between $f_{1}$ ' and the empirical photometric performance when the latter was calculated using 9 blackbody illuminants (top left), 9 fluorescent-based white LEDs (bottom left), 3 RGB LEDs (top right), and 9 blackbody illuminants together with 9 phosphor-based LEDs (bottom right) 
In Figure 2, we can see on the top left plot that $f_{1}$ " describes quite well the empirical photometric performance $\left\langle\delta s_{V}\right\rangle$ when measuring only blackbody illuminants, improving considerably the prediction capacity of the accepted index $f_{1}{ }^{\prime}$ (see top left plot in Figure 3 ). The correlation is not so good for phosphor-based LEDs (bottom left plot), although it is still much better than with $f_{1}{ }^{\prime}$ (compare with bottom left plot in Figure 3 ).

$f_{1}^{\prime \prime}$ for P15SOT is specially underestimated, if we compared this value with $\left\langle\delta s_{V}\right\rangle$. The result for this photometer worsens notably the correlation. The correlation of $f_{1}$ " with the empirical performance when evaluating the set with both blackbody illuminants and phosphor-based LEDs (bottom right plot in Figure 2) is slighter better to that obtained when evaluating only phosphor-based LEDs, and definitively much better than for $f_{1}$ ' (see bottom right plot in Figure 3). It must be noticed that for RGB LEDs $f_{1}$ " describes much worse the photometric performance than $f_{1}^{\prime}$ (compare top right plots in Figure 2 and Figure 3 ), which means that $f_{1}^{\prime}$ is adequate for quantifying photometric performance in the case of illuminants with narrow spectral distributions, where spectral response compensation unlikely applies, whereas $f_{1}{ }^{\prime \prime}$ is better for illuminants with broad spectral distributions.

If we examine the data at the bottom right plots in Figure 2 and Figure 3, for the complete set $B B+F L E D$ and for the seven high quality photometers of known spectral responsivity that we have used in this study, the correlation coefficient between $f_{1}$ ' and $\left\langle\delta s_{V}\right\rangle$ is -0.06 (completely uncorrelated), whereas between $f_{1}{ }^{\prime \prime}$ and $\left\langle\delta s_{V}\right\rangle$ is 0.82 , with a p-value (probability of getting a correlation as large as the observed value by random chance, when the true correlation is zero) of just $2.3 \%$.

\section{Conclusions}

It has been proven that a low value of $f_{1}$ is not enough to ensure a good photometric performance of a photometer, but that it also depends on the distribution of the spectral variation between the spectral responsivity of the photometer and $V(\lambda)$. An alternative general $V(\lambda)$ mismatch index, based on distribution of the spectral variation of the spectral responsivity with respect to $V(\lambda)$, is proposed. To compare both indices, it has been calculated the empirical photometric performance of seven photometers using a set of illuminants composed of 9 blackbody illuminants and 9 phosphor-based white LEDs. Whereas the values of the accepted general $V(\lambda)$ mismatch index $f_{1}{ }^{\prime}$ of the seven photometers is completely uncorrelated with their empirical performance, the proposed alternative index $f_{1}$ " is clearly correlated, except for the case of narrow band LED sources. Therefore, we consider that the use of this new index for the assessment of the general performance of the photometers would contribute in great extent in the general improvement of the photometric measurements.

\section{References}

CIE 2013. CIE S 023/E:2013. Characterization of the Performance of Illuminance Meters and Luminance Meters. Vienna: CIE.

CIE 2004. CIE S 010:2004. Photometry - The CIE system of physical photometry. Vienna: CIE.

ANSI 2015. ANSI C78.377-2015, American National Standard for Electric Lamps Specifications for the Chromaticity of Solid-state Lighting Products (NEMA). 